\author{
Mohammad MOMENIKIYAI, M.S. Student \\ E-mail:momenkiyai@yahoo.com \\ Department of Industrial Engineering, Karaj Branch, Islamic Azad \\ University, Karaj, Iran \\ Associate Professor Sadoullah EBRAHIMNEJAD *, PhD \\ E-mail: ibrahimnejad@kiau.ac.ir \\ Department of Industrial Engineering, Karaj Branch, Islamic Azad \\ University, Karaj, Iran \\ Assistant Professor Behnam VAHDANI, PhD \\ E-mail: b.vahdani@gmail.com \\ Department of Industrial Engineering, Faculty of Industrial and \\ Mechanical Engineering, Qazvin Branch, Islamic Azad University \\ Qazvin, Iran
}

\title{
A BI-OBJECTIVE MATHEMATICAL MODEL FOR INVENTORY- DISTRIBUTION-ROUTING PROBLEM UNDER RISK POOLING EFFECT: ROBUST META-HEURISTICS APPROACH
}

\begin{abstract}
In this paper, a bi-objective model is proposed to study LocationRouting-Inventory (LRI) problem considering risk pooling and soft time window. The first objective function aims to minimize the costs of establishing Distribution Centers $(D C s)$, transportation, ordering, and inventory holding; the second one minimizes the earliness and lateness of vehicles. Considering the large-scale instances of this problem is NP-Hard, three meta-heuristic algorithms, such as NSGA-II, MOPSO, and Pareto Envelope-based Selection Algorithm (PESA)-II, are proposed. These algorithms are compared, and NSGA-II outperforms the other algorithms. Afterward, the proposed NSGA-II is compared with the exact method. The computational results show that the exact method only outperforms this algorithm with around 6\% gap in the first objective function on average. Eventually, a sensitivity analysis has been conducted with respect to the number of DCs and vehicles. This shows that total costs, and total earliness and lateness increase and decrease in both cases, respectively.

Keywords: Risk Pooling; Vehicle Routing Problem; Location-Routing-Inventory Problem; NSGA II.

JEL Classification: C6 ,C61, C63, L81, M11, G31, G32
\end{abstract}

DOI: $10.24818 / 18423264 / 52.4 .18 .17$ 
Mohammad Momenikiyai, Sadoullah Ebrahimnejad, Behnam Vahdani

\section{Introduction}

In recent decades, competition, efficiency and effectiveness have been drawn the attention of practitioners due to globalization, increment in the diversity of goods and complexity of organizations.

Supply risk can affect differently the inventory management policies. SC risk management has been widely investigated since the concept of uncertainty was introduced into inventory theory. Uncertainty in SCs could be defined as both demand and supply uncertainties (Schmitt et al., 2015).

To solve uncertainty demand problem that leads to lost sales and holding SS, inventory aggregation or risk pooling is suggested which can decrease SS. Gaur and Ravindran (2006) stated that risk pooling is an efficient approach to decrease the SS, and consequently, to decrease inventory over the supply chain. Furthermore, they indicated that risk polling is an efficient approach when holding SS creates a large portion of supply chain costs.

The optimal allocation of vehicles for distribution decreases the transportation costs, and earliness and lateness. In such cases, increasing the lateness in delivering products could even force customers to choose one another products in the market. In numerous industries, such as food, pharmaceutical, and meat industries, holding goods more than sufficient will increase the holding costs. Finally, increasing earliness and lateness of products in such industries will also increase the dissatisfaction of customers, significantly. Thus, both of Risk pooling problem and Vehicle Routing Problem with Time Window (VRPTW) are studied, simultaneously.

The rest of this paper is organized as follows. The problem is explained in Section 3. The mathematical formulation is presented in Section 4. The mentioned meta-heuristic algorithms are proposed in Section 5. Section 6 presents computational results, and Section 7 provides a conclusion.

\section{Literature Review}

Gaur and Ravindran (2006) studied the effect of risk pooling on the supply chain. They stated that SS would decrease by risk pooling that depends on the correlation of retailers' and customers' demand. Ahmadi-Javid and Azad (2010) proposed a mathematical model to study LRI problem. They considered that demand of customers is uncertain and follows the normal distribution. They also assumed that each of retailers could hold SS. Ahmadi-Javid and Seddighi (2012) investigated the problem of LRI. They considered multiple cooperating suppliers in a three-echelon distribution 
A Bi-Objective Mathematical Model for Inventory-Distribution-Routing Problem Under Risk Pooling Effect: Robust Meta-Heuristics Approach

network. To solve large-scale instances, they proposed a meta-heuristic consisting of simulated annealing and ant colony algorithms.

Vahdani et al. (2017) proposed an integrated model to study the problem of Production-Inventory-Routing (PIR). They considered the limited capacity of vehicles and time window constraints for perishable products. Dehghani and Jabalameli (2017) investigated LRI problem under uncertainty. To deal with uncertainty, they used parameters of continuous Markov process. They tried to minimize the costs of location, distribution, and inventory. Nekooghadirli et al. (2014) proposed a biobjective mathematical model for the problem of LRI. They considered this problem for a multi-period and multi-product system. The first objective function minimizes the total, costs and the second one minimizes the average delivery time to costumers.

Schmitt et al. (2015) compared the centralization and decentralization of inventory with each other. They also reviewed different types of possible risks and disruptions threatening supply chain. Park et al. (2010) studied a three-echelon the supply chain considering inventory risk pooling and delivery time. Kang and Kim (2012) studied the problem of inventory control in a supply chain including one supplier, one DC, several regional depots, and several costumers. They proposed a mathematical model to minimize the total operational, inventory holding, and distribution costs. Tavakkoli-Moghaddam et al. (2013) proposed a bi-objective mathematical model to investigate LRI problem with risk pooling. The first objective function minimizes the total cost of the system, and the second one minimized the distribution time of goods.

Kumar and Tiwari (2013) proposed a mathematical model for the LRI problem with risk pooling. They were looking forward to finding the effect of risk pooling on the circulating inventory and SS level. Zhang et al. (2016) studied location of facilities problem considering the disruptions of facilities. When a facility confronts with disruption, customers' demand will be satisfied by other working facilities to avoid the lost sale.

Based on the literature, researchers published many papers for Vehicle Routing Problem (VRP) when customers' demand are satisfied within a specific interval of time (i.e., VRP with time window) (Corne et al., 2001). Considering Zhong and Cole (2005) and Low et al. (2013), most of the researchers investigated two types of time windows. In hard time window, customers' demand is only satisfied within the predefined interval of time. On the other hand, delivery time can take place even before or after this predefined interval of time in the soft time window. However, early 
Mohammad Momenikiyai, Sadoullah Ebrahimnejad, Behnam Vahdani

or late delivering of customers' demand happen before or after that predefined interval of time faces with a penalty added to the objective function in the soft time window (Yan et al., 2015).

In this paper, the problem of LRI with risk pooling is considered and a biobjective mathematical model is proposed. In contrast to previous investigations, the main contribution of this paper is threefold:

- Total earliness and lateness are considered as an objective function.

- Fleet is considered to be heterogeneous.

- Three meta-heuristic algorithms have been developed and compared with each other.

\section{Problem statement}

To satisfy the demand of customers, two following cases are possible:

- Satisfy customers' demand by their specific depots

- Satisfy customers' demand by only a specific depot

In the first case, each depot holds its required SS. This will increase the total SS held in the supply chain. In the second case, the demand of customers is aggregated in one depot. This helps to decrease the uncertainty existing in demand, and consequently, to decrease the SS. Inventory aggregation (also called risk pooling) is an efficient approach to decrease the SS and to decrease total inventory throughout the supply chain (Gaur and Ravindran, 2006).

In the case of centralized SS, required SS is determined based on Eppen (1979), and Chen and Lin (1989)

While in the case of decentralized SS is implemented, required SS is determined based on Kumar and Tivari, (2012).

\section{Mathematical formulation} considered:

To formulate the problem mentioned above, following assumptions are

- A two-echelon supply chain that includes DCs and customers and the demand of customers is uncertain and follows normal distribution function, and is independent.

- Establishing cost of DCs depend on the capacity of them.

- All DCs have specific fixed costs of ordering and inventory holding. 
A Bi-Objective Mathematical Model for Inventory-Distribution-Routing Problem Under Risk Pooling Effect: Robust Meta-Heuristics Approach

- $\quad$ DC $j$ follows the inventory policy of $\left(Q_{j}, R_{j}\right)$. Furthermore, each of DCs can hold SS; furthermore, fixed order quantity $Q$ is purchased when inventory level reaches to reorder point $R$.

- Transportation fleet is heterogeneous.

- Transportation time between nodes is deterministic.

Considering above-mentioned assumptions, we define the following indices, parameters, and decision variables for the mathematical formulation.

\section{- Indices and sets:}

$k \quad$ Index of customers $\quad k \in\{1,2, \ldots, K\}$

$j \quad$ Index of potential DCs $\quad j \in\{1,2, \ldots, J\}$

$v \quad$ Index of vehicle $\quad v \in\{1,2, \ldots, V\}$

$n \quad$ Index of DCs' capacity $n \in\left\{1,2, \ldots, N_{j}\right\}$

$M \quad$ Union of customers' and DCs' sets (i.e., $K \cup J$ )

\section{- Parameters:}

$B \quad$ Number of all customers (i.e., $B=|K|$ )

$\mu_{k} \quad$ Average annual demand of customer $k$

$\sigma_{k}^{2} \quad$ Variance of annual demand of customer $k$

$f_{j}^{n} \quad$ Total operational cost and establishing cost of DC $j$ with capacity $n$

$b_{j}^{n} \quad$ Capacity with level $n$ for DC $j$

$d_{k l} \quad$ Transportation cost between nodes $k$ and $l$

$v c_{v} \quad$ Annual delivery capacity of vehicle $v$

$q \quad$ Number of times that each of customers is visited within a year

$h_{j} \quad$ Annual cost of inventory holding for DCj (per each unit product)

$p_{j} \quad$ Fixed ordering cost for DC $j$

$l t_{j} \quad$ Lead time of DC $j$ in year

$g_{j} \quad$ Fixed cost per shipment from supplier to $\mathrm{DC} \mathrm{j}$

$a_{j} \quad$ Shipment cost from supplier to $\mathrm{DC} j$ (per each unit product)

$\operatorname{Pr}_{v} \quad$ Fixed cost of using vehicle $v$

$\alpha \quad$ Desired percentage of customer orders that should be satisfied

$z_{\alpha} \quad$ Left a-percentile of standard normal random variable $\mathrm{Z}$

$\beta \quad$ Weight factor associated with transportation cost

$\theta \quad$ Weight factor associated with inventory cost

$s_{k}^{v} \quad$ Service time of customer $k$

$t_{k l}^{v} \quad$ Travel time from node $k$ to node $l$ by vehicle $v$ 
Mohammad Momenikiyai, Sadoullah Ebrahimnejad, Behnam Vahdani

$e_{k} \quad$ Lower bound of time window for customer $k$

$l_{k} \quad$ Upper bound of time window for customer $k$

$H \quad$ An adequately large number

\section{- Decision variables:}

$R_{k l v} \quad$ If vehicle $v$ travels from node $k$ to node $l 1$; otherwise, 0 .

$Y_{j k} \quad$ If Customer $k$ is assigned to $\mathrm{DC} j 1$; otherwise, 0 .

$U_{j}^{n} \quad$ If DC $j$ is established with capacity $n 1$; otherwise,

$E_{k}^{v} \quad$ Earliness of vehicle $v$ for customer $k$

$L_{k}^{v} \quad$ Lateness of vehicle $v$ for customer $k$

$m_{k}^{v} \quad$ Arrival time of vehicle $v$ to customer $k$

$M_{k v} \quad$ An auxiliary variable to eliminate sub-tours

The proposed model includes two objective functions; these objective functions are formulated as follows:

$$
\begin{aligned}
& \operatorname{Min}_{1}=\sum_{j \in J} \sum_{n \in N_{j}} f_{j}^{n} U_{j}^{n}+\beta q\left(\sum_{v \in V} \sum_{k \in M} \sum_{l \in M} d_{k l} R_{k l v}+\sum_{v \in V} \sum_{j \in J} \sum_{l \in M} P r_{v} R_{j l v}\right)+ \\
& \sum_{j \in J}\left[\left(\theta p_{j}+\beta g_{j}\right) \frac{\sum_{k \in K} \mu_{k} Y_{j k}}{Q_{j}}+\beta a_{j} \sum_{k \in K} \mu_{k} Y_{j k}+\theta h_{j} z_{\alpha} \sqrt{l t_{j} \sum_{k \in K} \sigma_{k}^{2} Y_{j k}}\right]
\end{aligned}
$$

The first objective function minimizes the fixed costs of establishing DCs, annual costs of transportation and inventory costs. To be more precise, the first and second terms minimize the fixed costs of establishing DCs, and annual costs of transportation, respectively. The third term also minimizes the inventory costs.

$\operatorname{Min} f_{2}=\sum_{v \in V} \sum_{j \in J}\left(E_{j}^{v}+L_{j}^{v}\right)$

The second objective function also minimizes the earliness and lateness in satisfying customers' demand.

The derivative of the first objective function regarding $Q_{j}$ is equal to Eq. (3). we replace $Q_{j}$ by Eq. (3), in Eq. (4).

$$
\begin{aligned}
& Q_{j}^{*}=\sqrt{\frac{2 \theta h_{j}\left(\theta p_{j}+\beta g_{j}\right) \sum_{k \in K} \mu_{k} Y_{j k}^{2}}{\theta h_{j}}} \\
& M i n f_{1}=\sum_{j \in J} \sum_{n \in N_{j}} f_{j}^{n} U_{j}^{n}+\beta q\left(\sum_{v \in V} \sum_{k \in M} \sum_{l \in M} d_{k l} R_{k l v}+\sum_{v \in V} \sum_{j \in J} \sum_{l \in M} P r_{v} R_{j l v}\right)+
\end{aligned}
$$


A Bi-Objective Mathematical Model for Inventory-Distribution-Routing Problem Under Risk Pooling Effect: Robust Meta-Heuristics Approach

$\sum_{j \in J}\left[\sqrt{2 \theta h_{j}\left(\theta p_{j}+\beta g_{j}\right) \sum_{k \in K} \mu_{k} Y_{j k}}+\beta a_{j} \sum_{k \in K} \mu_{k} Y_{j k}+\theta h_{j} z_{\alpha} \sqrt{l t_{j} \sum_{k \in K} \sigma_{k}^{2} Y_{j k}}\right]$

The continuous relaxation of the first objective function (i.e., Eq. (1)) is concave. Therefore, reaching the global optimum solution is not guaranteed using the existing methods. Since $Y_{j k}$ is a binary variable, we will replace $Y_{j k}$ by $Y_{j k}^{2}$ in the first objective function to convexity. Eventually, the first objective function is formulated as Eq. (5) (Ahmadi-Javid and Azad, 2010).

$$
\begin{aligned}
& \operatorname{Min}_{1}=\sum_{j \in J} \sum_{n \in N_{j}} f_{j}^{n} U_{j}^{n}+\beta q\left(\sum_{v \in V} \sum_{k \in M} \sum_{l \in M} d_{k l} R_{k l v}+\sum_{v \in V} \sum_{j \in J} \sum_{l \in M} P r_{v} R_{j l v}\right)+ \\
& \sum_{j \in J}\left[\sqrt{2 \theta h_{j}\left(\theta p_{j}+\beta g_{j}\right) \sum_{k \in K} \mu_{k} Y_{j k}^{2}}+\beta a_{j} \sum_{k \in K} \mu_{k} Y_{j k}^{2}+\theta h_{j} z_{\alpha} \sqrt{l t_{j} \sum_{k \in K}{\sigma_{k} Y_{j k}}^{2}}\right]
\end{aligned}
$$

These objective functions are subjected to the following constraints:

$$
\begin{array}{ll}
m_{k}^{v}+s_{k}^{v}+t_{k l}^{v}-m_{l}^{v} \leq\left(1-R_{k l v}\right) H & \forall v \in V . \forall k . l \in M . k \neq l \\
E_{k}^{v} \geq e_{k} \sum_{l \in M} R_{k l v}-m_{k}^{v} & \forall k \in M . v \in V \\
L_{k}^{v} \geq m_{k}^{v}-l_{k} & \forall k \in M . v \in V \\
\sum_{v \in V} \sum_{l \in M} R_{k l v}=1 & \forall k \in K \\
\sum_{l \in K} \mu_{l} \sum_{k \in M} R_{k l v} \leq v c_{v} & \forall v \in V \\
M_{k v}-M_{l v}+\left(B \times R_{k l v}\right) \leq B-1 & \forall k . l \in K . \forall v \in V \\
\sum_{l \in M} R_{k l v}-\sum_{l \in M} R_{l k v}=0 & \forall k \in M . \forall v \in V \\
\sum_{j \in J} \sum_{k \in K} R_{j k v} \leq 1 & \forall v \in V \\
\sum_{l \in M} R_{k l v}+\sum_{l \in M} R_{j l v}-Y_{j k} \leq 1 & \forall j \in J . \forall k \in K . \forall v \in V \\
\sum_{n \in N_{j}} U_{j}^{n} \leq 1 & \forall j \in J \\
\sum_{k \in K} \mu_{k} Y_{j k} \leq \sum_{n \in N_{j}} b_{j}^{n} U_{j}^{n} & \forall j \in J
\end{array}
$$


Mohammad Momenikiyai, Sadoullah Ebrahimnejad, Behnam Vahdani

$$
\begin{aligned}
& \overline{\sum_{v \in V} m_{j}^{v}=0} \\
& Y_{j k}, U_{j}^{n}, R_{k l v} \in\{0.1\} \\
& E_{k}^{v} \cdot L_{k}^{v} \cdot m_{k}^{v}, M_{k v} \geq 0
\end{aligned}
$$

Constraint (6) determines the arrival time of vehicles. Constraints (7) and (8) calculate the earliness and lateness in serving customer, respectively. Constraint (9) assures that the demand of each customer is satisfied by only one vehicle. Constraint (10) refers to the limited capacity of vehicles. Constraint (11) eliminates sub-tours. In the other words, this constraint ensures that each tour consists of merely one DC and some customers. Constraint (12) ensures that vehicles enter to a node must leave this node. Constraint (13) ensures that only one DC is included in each tour. Constraint (14) indicate that customer $k$ is assigned to DC $j$ if there is a tour that starts from this DC and contains node $k$. Constraint (15) guarantees that establishing each of DCs is possible with a specific capacity level. Constraint (16) refers to the limited capacity of DCs. Constraint (17) assures that tours start at the beginning of planning horizon. Finally, Constraints (18) and (19) determine binary and positive variables of the proposed model, respectively.

\section{The proposed meta-heuristic algorithms}

For solve proposed model, we used NSGA-II, MOSPO and PESA-II. For more details see (Deb et al. 2000), (Deb et al., 2002) and (Coello et al., 2004).

\section{Solution representation and initial solution}

Since a solution to this problem consists of multiple decision variables, we will illustrate the representation of each decision variable separately for more clarification.

Number of open DCs: If we considerJDCs and $n$ potential capacity levels, a $J$ by $n+1$ matrix is randomly generated that contains real numbers between zero and one. Noted that these numbers are generated based on a uniform distribution function (this holds in the rest of this paper). The first column determines which DC is whether open or not. For this purpose, we set the value of the first column equal to one where the generated random values are greater than or equal to 0.5 . On the other hand, if these values are less than 0.5 , we set them equal to zero. If the value mapped to the first column and row $j$ is equal to one, we consider that the DCjis open; otherwise, it is close. Afterward, we will find the rows that their first column is equal to one (i.e., open DCs). The largest number mapped to each of these rows (except their first column) is selected and is set equal to one; then, the 
A Bi-Objective Mathematical Model for Inventory-Distribution-Routing Problem Under Risk Pooling Effect: Robust Meta-Heuristics Approach

other columns of these rows are set equal to zero. Furthermore, the value of other rows, where the DC is close, is set equal to zero. Fig. (1) shows an illustrative example (an example with three potential DCs and three potential capacity levels) for this section of the solution representation.

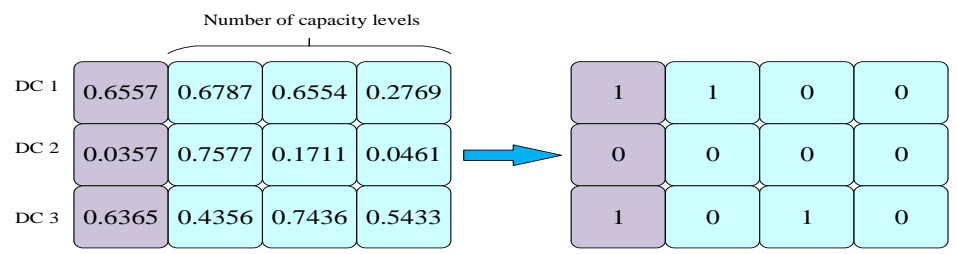

Figure 1.An illustrative example for number of opened DCs

Assignment of customers to DCs: If we consider JDCs and $B$ customers, a $J$ by $B$ matrix is randomly generated that contains real numbers between zero and one. If DC $j$ is not open based on the previous step, we will set all the elements of row $j$ equal to zero. Then, we have to recognize the largest value for each of columns (i.e., each of customers), and set it equal to one. Finally, we will set the rest of values in each of columns equal to zero. Fig. (2) shows an illustrative example (an example with two potential DCs and four customers) for this section of the solution representation.

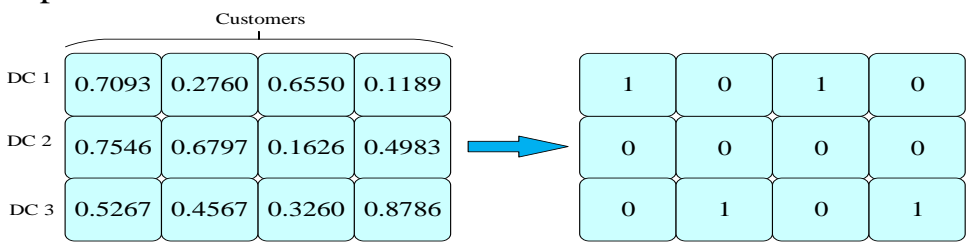

Figure 2.An illustrative example for assignment of customers to DCs

Assignment of vehicles to DCs: If we consider JDCs and $V$ vehicles, a $J$ by $V$ matrix is randomly generated that contains real numbers between zero and one. If DC $j$ is not open, we will set all the values of row $j$ equal to zero. To assign vehicles to DCs, we have implemented a two-step procedure as follows. Firstly, one vehicle is assigned to each DC. To do so, the largest value for each of rows (i.e., each of DCs) are recognized and set equal to one. Then, the rest of values in the column of selected value are set equal to zero to ensure that no vehicles have been assigned to more than one DCs, simultaneously. Secondly, the rest of vehicles, which are not allocated to DCs, will be assigned. For this purpose, the 
Mohammad Momenikiyai, Sadoullah Ebrahimnejad, Behnam Vahdani

largest number in each of these columns are recognized and allocated to the corresponding DC. Fig. (3) shows an illustrative example (an example with two potential DCs and four customers) for this section of the solution representation.

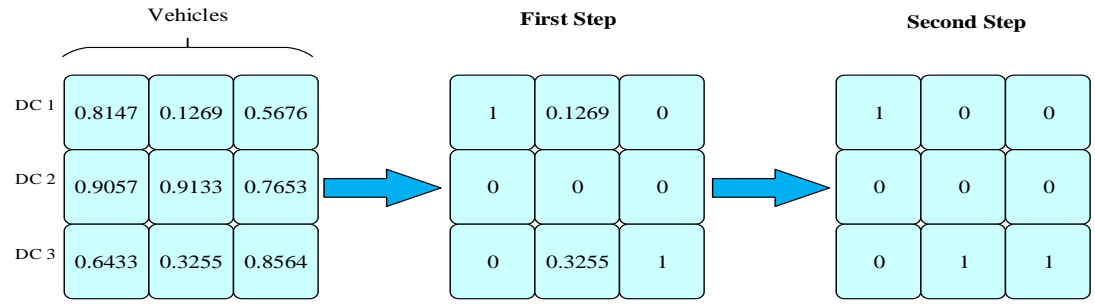

Figure 3.An illustrative example for assignment of vehicles to DCs

Assignment of vehicles to customers: If we consider $B$ customers and $V$ vehicles, a $B$ by $V$ matrix is randomly generated that contains real numbers between zero and one. If $a_{k v}$ refers to the element of row $k$ and column $v$, we assign each of customers to each of vehicles. First, if customer $k$ is not assigned to DC $j$, and furthermore, vehicle $v$ is not assigned to DC $j$, we will set $a_{k v}$ equal to zero. Then, we will find the largest value in each row and set it equal to one; we will set the rest elements of all rows equal to zero. Fig. (4) shows an illustrative example (an example with four customers and two vehicles) for this section of the solution representation.

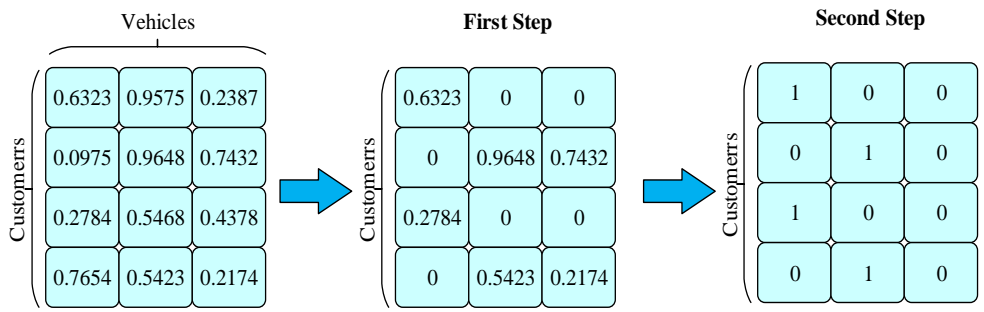

Figure 4. An illustrative example for assignment of vehicles to customers

The sequence of customers assigned to routes: If we consider $B$ customers, a one by $B$ matrix is randomly generated that contains real numbers between zero and one. Then, all elements are sorted in ascending order to determine the sequence of serving customers. Fig. (5) shows an illustrative example (an example with four customers) for this section of the solution representation. 
A Bi-Objective Mathematical Model for Inventory-Distribution-Routing Problem Under Risk Pooling Effect: Robust Meta-Heuristics Approach

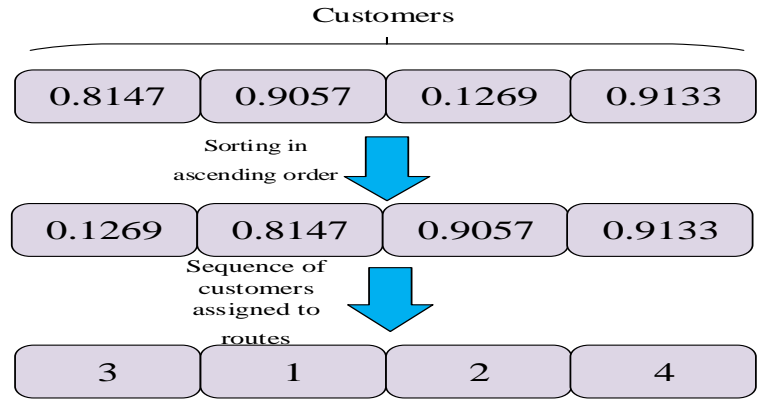

Figure 5.An illustrative example for sequence of customers assigned to routes

\section{Crossover and Mutation}

Crossover and mutation are two operators that mostly used in meta-heuristic algorithms to produce high-quality solutions, and increase the solutions' diversity, respectively. We refer interested readers toSoni and Kumar (2014) for further detail to seek for brevity.

\section{Penalty Function}

The structures introduced in previous sub-sections cannot guarantee the feasibility of solution regarding the Constraints (10) and (16). For this purpose, the average value of these violations is calculated based on Eqs. (21) and (23) an added to the first objective function to form Eq. (24):

$V D C_{j}=\max \left(\frac{D C_{j}}{T D C}-1,0\right)$

Mean_VDC $=\operatorname{mean}\left(V D C_{j}\right)$

$V V_{v}=\max \left(\frac{V_{v}}{T V}-1,0\right)$

$M e a n_{-} V V=\operatorname{mean}\left(V V_{v}\right)$

$f_{1}^{\prime}=f_{1} *\left(1+b e t a *\left(M e a n_{-} V D C+M e a n_{-} V V\right)\right)$

where $V D C_{j}$ and $V V_{v}$ refer to the capacity violation occurred for $\mathrm{DC} j$ and vehicle $v$, respectively. Furthermore, $D C_{j}$ and $V_{v}$ refer to the capacity of $\mathrm{DC} j$ and vehicle $v$, respectively; TDC and TV refer to the total capacity of DCs and vehicles, respectively. 
Mohammad Momenikiyai, Sadoullah Ebrahimnejad, Behnam Vahdani

It should be noted that beta in Eq. (24) refers to the magnifier of violations value and is set equal to 1000 .

\section{Computational results}

To generate instances randomly, we have defined an interval for each of parameters used in the proposed model. For this purpose, the range of parameters is provided in Table (1).

Table 1. Range of parameters used in the proposed model

\begin{tabular}{l|l|l|l}
\hline Parameter & Range & Parameter & Range \\
\hline$\mu_{k}$ & Uniform [400, 1500] & $a_{j}$ & Uniform [5, 10] \\
$\sigma_{k}^{2}$ & Uniform [10,30] & $\theta$ & 0.7 \\
$d_{k l}$ & Uniform [0,300] & $\beta$ & 0.3 \\
$h_{j}$ & Uniform [5, 10] & $s_{k}^{v}$ & Uniform [1, 48] \\
$p_{j}$ & Uniform [10, 15] & $t_{k l}^{v}$ & Uniform [0.5, 5] \\
$l t_{j}$ & Uniform [6/365, 10/365] & $e_{k}$ & Uniform [8, 20] \\
$g_{j}$ & Uniform [10,15] & $l_{k}$ & $e_{k}+$ Uniform [1, 6] \\
\hline
\end{tabular}

It is assumed that a year consists of 300 days and each of vehicles will visit customers every three days. Thus, $q$ is equal to 100 . Since the satisfaction rate of customers' demand is set equal to $97.5 \%$, therefore, $Z_{\alpha}$ is equal to 1.96 .The capacity of vehicles is determined based on Eq. (25) in which $|V|$ and $D$ refer to the total number of vehicles and average demand of customers, respectively.

$v c_{v}=$ uniform $\left[2 \times\left[\frac{D}{|V|}\right], 3 \times\left[\frac{D}{|V|}\right]\right.$

The largest instance that the exact method could solve in rational CPU Time was an instance with six customers and two DCs. The Pareto solutions found using $\varepsilon$ constraint method is provided in Table (2) for the largest instance solved by the exact method. For more clarification, Fig. (6) illustrates the last Pareto solution provided in Table (2).

Table 2. The Pareto solutions obtained with the exact method using $\varepsilon$-constraint

\begin{tabular}{|c|c|c|c|c|c|c|c|c|c|c|c|c|c|c|c|}
\hline & $z_{1}$ & 2232.165 & 2232.165 & 2242.61 & 2264.092 & 2287.773 & 2307.075 & 2322.584 & 2863.444 & 2901.013 & 2916.619 & 2916.619 & 2964.688 & 2985.259 & 3029.494 \\
\hline$Z_{2}$ & Earliness (h) & 0 & 0 & 0 & 0 & 0 & 0 & 0 & 0 & 0 & 0 & 10 & 10 & 10 & 10 \\
\hline & Lateness (h) & 495 & 441 & 306 & 292 & 287 & 280 & 248 & 164 & 142 & 124 & 104 & 102 & 101 & 97 \\
\hline & Total & 495 & 441 & 306 & 292 & 287 & 280 & 248 & 164 & 142 & 124 & 114 & 112 & 111 & 107 \\
\hline
\end{tabular}


A Bi-Objective Mathematical Model for Inventory-Distribution-Routing Problem Under Risk Pooling Effect: Robust Meta-Heuristics Approach

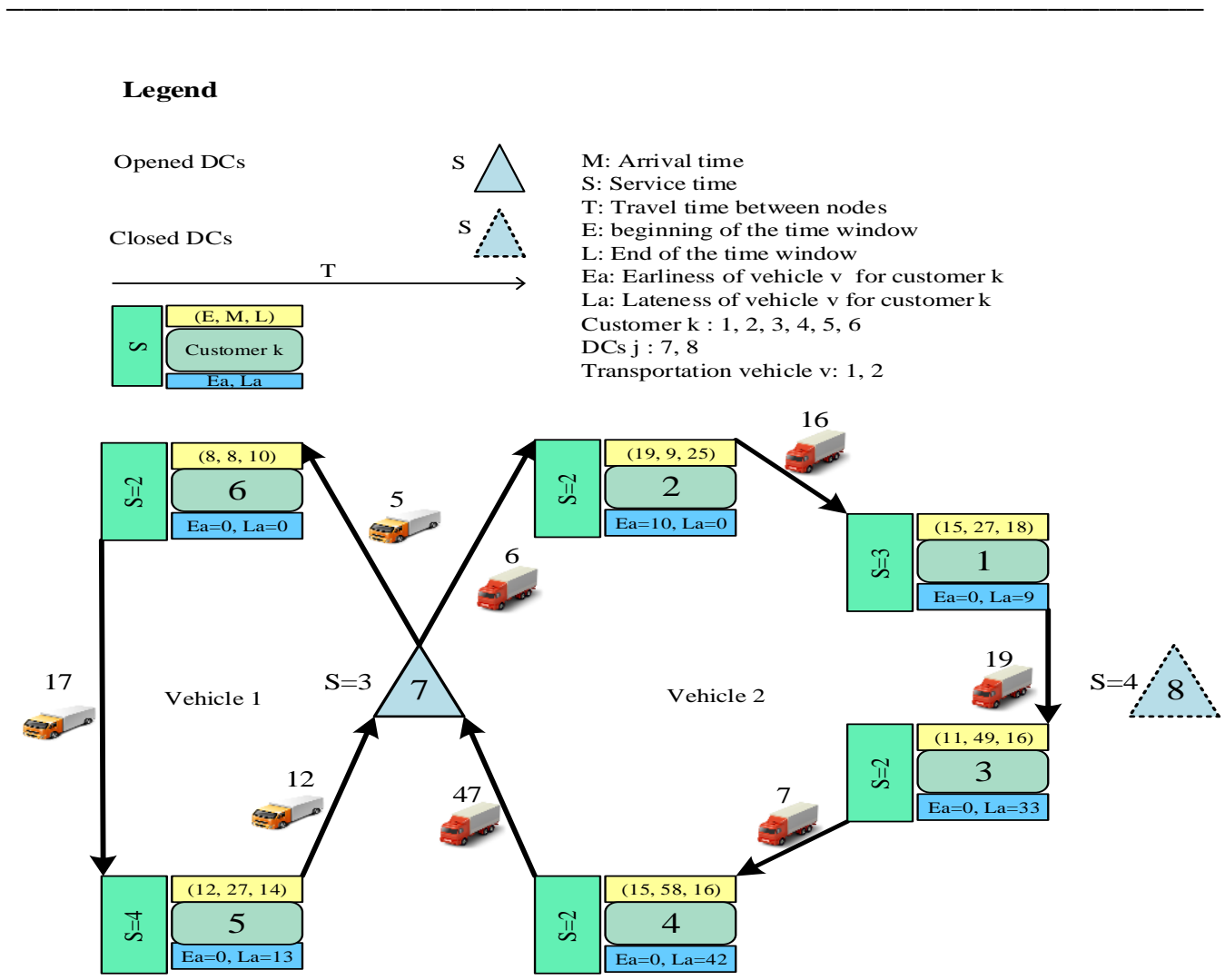

Figure 6. A schema for the largest instances solved with the exact method

Therefore, it is obvious that proposing meta-heuristic algorithm was essential.

\subsection{Parameters of the proposed algorithms}

In order to compare the quality of non-dominated solutions, four criteria such as Quality Metric (QM), Mean Ideal Distance (MID), Diversification Metric (DM) and Spacing Metric (SM) are used. For more details, see Moradi et al. (2011) and Nekooghadirli et al. (2014).

Taguchi experimental design method has been applied in order to determine the optimal level of parameters in NSGA-II, MOPSO and PESA-II algorithms. For this purpose, the Parameters of these algorithms are designed in three High, Medium and Low level. Then, the optimal setting for the parameters of these algorithms is 
Mohammad Momenikiyai, Sadoullah Ebrahimnejad, Behnam Vahdani

determined and used in solving large scale instances in order to increase the quality of solutions.

\subsection{Comparison of the proposed algorithms}

To compare the proposed algorithms, 30 instances are randomly generated based on the data provided in Table (1). Then, all of the instances are solved using three proposed algorithms.

Considering 12t-paired tests (four performance metrics and three algorithms) the proposed NSGA-II provides better solutions with respect to MID and DM. Furthermore, the proposed NSGA-II and the proposed PESA-II outperform the proposed MOPSO in terms QM. But the proposed NSGA-II can not only provide better solution compared to two other algorithms regarding SM. Therefore, it can be argued that the proposed NSGA-II outperforms two other algorithms.

\subsection{Comparison of the proposed NSGA-II and exact method}

Since the proposed NSGA-II outperforms other meta-heuristic algorithms, we compared the computational results of based on NSGA-II algorithm and the exact method in an instance with six customers, two distributions centers, and two vehicles in Table (3).

Table 3. Comparison the Pareto solutions provided by the proposed NSGA-II and $\varepsilon$-constraint method

\begin{tabular}{|c|c|c|c|c|c|c|c|c|c|}
\hline \multicolumn{4}{|c|}{ The proposed NSGA-II } & \multicolumn{4}{|c|}{$\varepsilon$-constraint method } & \multirow{2}{*}{\multicolumn{2}{|c|}{ Gap (\%) }} \\
\hline \multirow{2}{*}{$\begin{array}{l}\text { Cost } \\
- \\
\text { Obj1 }\end{array}$} & \multicolumn{3}{|c|}{$\begin{array}{l}\text { Earliness and lateness - } \\
\text { Obj2 (hour) }\end{array}$} & \multirow[t]{2}{*}{$\begin{array}{l}\text { Cost - } \\
\text { Obj1 }\end{array}$} & \multicolumn{3}{|c|}{$\begin{array}{l}\text { Earliness and lateness - } \\
\text { Obj2 (hour) }\end{array}$} & & \\
\hline & Total & Earliness & Lateness & & Total & Earliness & Lateness & Obj1 & Obj2 \\
\hline 2347 & 495 & 0 & 495 & 2232.165 & 495 & 0 & 495 & 5.14 & 0.00 \\
\hline 2364 & 441 & 0 & 441 & 2232.165 & 441 & 0 & 441 & 5.91 & 0.00 \\
\hline 2374 & 306 & 0 & 306 & 2242.61 & 306 & 0 & 306 & 5.86 & 0.00 \\
\hline 2393 & 292 & 0 & 292 & 2264.092 & 292 & 0 & 292 & 5.69 & 0.00 \\
\hline 2419 & 287 & 0 & 287 & 2287.773 & 287 & 0 & 287 & 5.74 & 0.00 \\
\hline 2436 & 280 & 0 & 280 & 2307.075 & 280 & 0 & 280 & 5.59 & 0.00 \\
\hline 2454 & 248 & 0 & 248 & 2322.584 & 248 & 0 & 248 & 5.66 & 0.00 \\
\hline 3033 & 164 & 0 & 164 & 2863.444 & 164 & 0 & 164 & 5.92 & 0.00 \\
\hline 3066 & 142 & 0 & 142 & 2901.013 & 142 & 0 & 142 & 5.69 & 0.00 \\
\hline 3066 & 124 & 0 & 124 & 2916.619 & 124 & 0 & 124 & 5.12 & 0.00 \\
\hline 3083 & 114 & 10 & 104 & 2916.619 & 114 & 10 & 104 & 5.70 & 0.00 \\
\hline
\end{tabular}


A Bi-Objective Mathematical Model for Inventory-Distribution-Routing Problem Under Risk Pooling Effect: Robust Meta-Heuristics Approach

\begin{tabular}{l|lll|l|lll|ll}
\hline 3131 & 112 & 10 & 102 & 2964.688 & 112 & 10 & 102 & 5.61 & 0.00 \\
\hline 3152 & 111 & 10 & 101 & 2985.259 & 111 & 10 & 101 & 5.59 & 0.00 \\
\hline 3173 & 107 & 10 & 97 & 3029.494 & 107 & 10 & 97 & 4.74 & 0.00 \\
\hline
\end{tabular}

As Table (3) shows, the results provided by the proposed NSGA-II is comparable with the ones provided by the exact method. To be more precise, the exact method outperforms the proposed NSGA-II with around $6 \%$ gap in only one the objective functions. It means that the solutions provided by the proposed NSGA-II are reliable.

\subsection{Sensitivity analysis}

In this subsection, a sensitivity analysis has been conducted to figure out how increment in the number of vehicles and potential DCs affect the solutions. For this purpose, we have studied Instance 23for both cases. In the first case, we have increased the number of DCs from 18 to 28 , while other parameters are fixed. Likewise, we have also increased the number of vehicles from 18 to 29 in the second case, while other parameters are fixed. To compare Pareto fronts obtained for each of these cases, one mediocre solution has been selected from each of these Pareto fronts. Then, they have been compared regarding first and second objective functions. Figs. (7) and (8) show the results of this sensitivity analysis when the number of DCs and vehicles have been increased, respectively. As shown in Fig. (7), increment in the number of DCs increases total costs. Furthermore, it decreases the distance between DCs and customers, and consequently, decreases total lateness and earliness. Fig. (8) shows also increment in the number of vehicles increases total costs, while decrease total earliness and lateness. In other words, increasing availability of vehicles decreases total lateness and earliness.

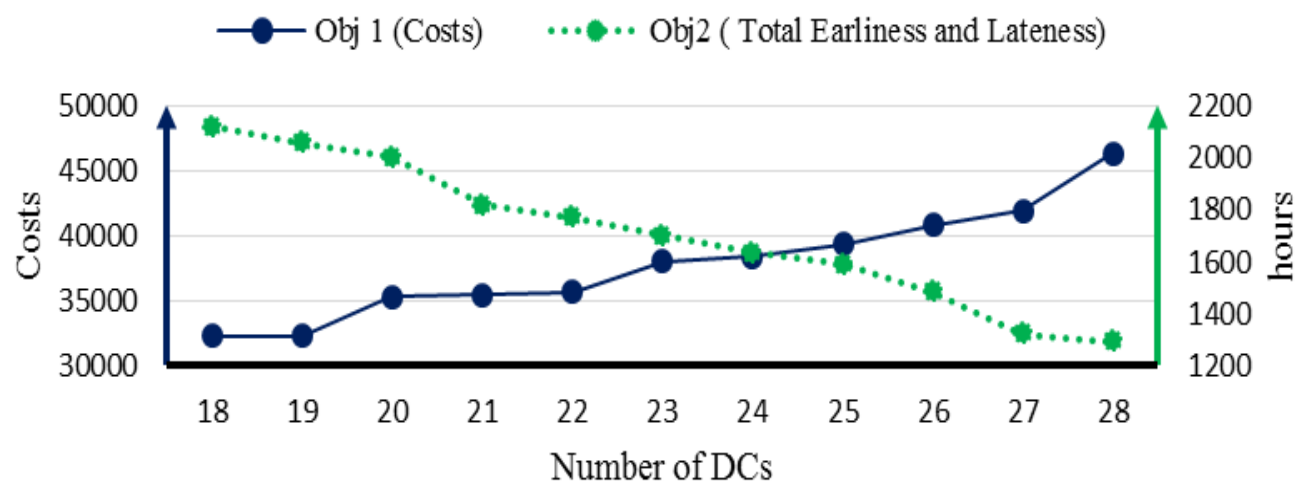

Figure 7. Sensitivity analysis for the number of DCs 
Mohammad Momenikiyai, Sadoullah Ebrahimnejad, Behnam Vahdani

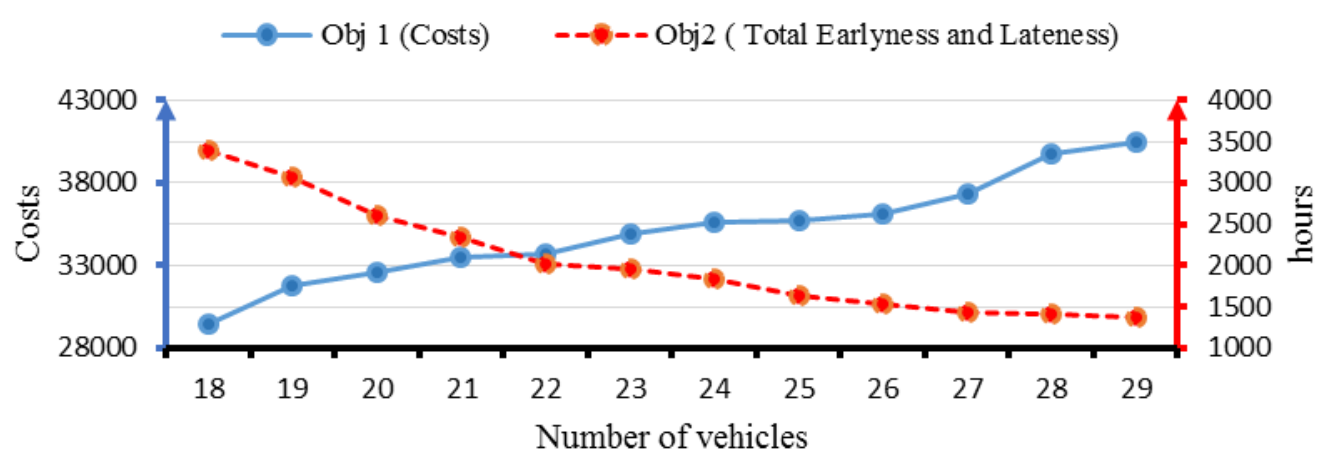

Figure 8. Sensitivity analysis for the number of vehicles

\section{Conclusion}

In this paper, a bi-objective mathematical model was proposed study LRI problem considering risk pooling and soft time window. The first objective function of the proposed model aims to minimize costs of establishing DCs, transportation, ordering, and inventory holding, and the second one minimizes the earliness and lateness of vehicles. Considering the large-scale instances of this problem is NP-Hard, three metaheuristic algorithms, such as NSGA-II, MOPSO, and PESA-II were proposed in this paper. To compare these algorithms, four well-known criteria were used. But before comparing the algorithms, some of theimportant parameters for each of these algorithms were determined. Then, three levels were determined, and the optimal combination of these parameters was determined by Taguchi experimental design method.

For future research, taking into account environmental objectives besides considering multi-product and multi-period mathematical model would be interesting.

\section{REFERENCES}

[1]Ahmadi-Javid, A. \& Seddighi, A. H. (2012), A Location-Routing-Inventory Model for Designing Multisource Distribution Networks. Engineering Optimization, 44(6), 637-656;

[2]Ahmadi-Javid, A. \& Azad, N. (2010), Incorporating Location, Routing and Inventory Decisions in Supply Chain Network Design. Transportation Research Part E: Logistics and Transportation Review, 46(5), 582-597; 
A Bi-Objective Mathematical Model for Inventory-Distribution-Routing Problem Under Risk Pooling Effect: Robust Meta-Heuristics Approach

[3]Chen, M.-S. \& Lin, C.-T. (1989),Effects of Centralization on Expected Costs in a Multi-Location Newsboy Problem. Journal of the Operational Research Society, 40(6), 597-602;

[4]Coello, C. A. C., Pulido, G. T. \& Lechuga, M. S. (2004), Handling Multiple

Objectives with Particle Swarm Optimization. IEEE transactions on Evolutionary Computation, 8(3), 256-279;

[5]Deb, K., Agrawal, S., Pratap, A. \& Meyarivan, T. (2000), A Fast Elitist NonDominated Sorting Genetic Algorithm for Multi-Objective Optimization: NSGA-II. Paper presented at the International Conference on Parallel Problem Solving From Nature;

[6]Deb, K., Pratap, A., Agarwal, S. \& Meyarivan, T. (2002), A Fast and Elitist Multiobjective Genetic Algorithm: NSGA-II. IEEE transactions on Evolutionary Computation, 6(2), 182-197;

[7]Dehghani, E. \& Jabalameli, M. S. (2017), Optimizing Location, Routing and Inventory Decisions in an Integrated Supply Chain Network Under Uncertainty. Journal of Industrial and Systems Engineering, 9(4), 93-111;

[8]Eppen, G. D. (1979), Note-Effects of Centralization on Expected Costs in a Multi-Location Newsboy Problem. Management Science, 25(5), 498-501;

[9]Gaur, S. \& Ravindran, A. R. (2006), A Bi-Criteria Model for the Inventory Aggregation Problem under Risk Pooling. Computers \& Industrial Engineering, 51(3), 482-501;

[10]Kang, J.-H. \& Kim, Y.-D. (2012), Inventory Control in a Two-Level Supply

Chain With Risk Pooling Effect. International Journal of Production Economics, 135(1), 116-124;

[11]Kumar, S. K. \& Tiwari, M. (2013), Supply Chain System Design Integrated with Risk Pooling. Computers \& Industrial Engineering, 64(2), 580-588;

[12]Low, C., Li, R.-K. \& Chang, C.-M. (2013), Integrated Scheduling of Production and Delivery with Time Windows. International Journal of Production Research, 51(3), 897-909;

[13]Moradi, H., Zandieh, M. \& Mahdavi, I. (2011), Non-dominated Ranked Genetic Algorithm for a Multi-Objective Mixed-Model Assembly Line Sequencing Problem. International Journal of Production Research, 49(12), 3479-3499; [14]Nekooghadirli, N., Tavakkoli-Moghaddam, R., Ghezavati, V. R., \& Javanmard, S. (2014), Solving a New Bi-Objective Location-Routing-Inventory Problem in a Distribution Network By Meta-Heuristics. Computers \& Industrial Engineering, 76, 204-221; 
Mohammad Momenikiyai, Sadoullah Ebrahimnejad, Behnam Vahdani

[15]Park, S., Lee, T.-E. \& Sung, C. S. (2010), A Three-level Supply Chain Network Design Model with Risk-Pooling and Lead Times. Transportation Research Part E: Logistics and Transportation Review, 46(5), 563-581.

[16]Schmitt, A. J., Sun, S. A., Snyder, L. V. \& Shen, Z.-J. M. (2015),Centralization versus Decentralization: Risk Pooling, Risk Diversification, and Supply Chain Disruptions. Omega, 52, 201-212;

[17]Soni, N. \& Kumar, T. (2014), Study of Various Mutation Operators in Genetic Algorithms. International Journal of Computer Science and Information Technologies, 5(3), 4519-4521;

[18]Tavakkoli-Moghaddam, R., Azarkish, M. \& Sadeghnejad-Barkousaraie, A. (2011), A New Hybrid Multi-Objective Pareto Archive PSO Algorithm for a BiObjective Job Shop Scheduling Problem. Expert Systems with Applications, 38(9), 10812-10821;

[19]Tavakkoli-Moghaddam, R., Forouzanfar, F. \& Ebrahimnejad, S. (2013), Incorporating Location, Routing, and Inventory Decisions in a Bi-Objective Supply Chain Design Problem with Risk-Pooling. Journal of Industrial Engineering International, 9(1), 19;

[20]Vahdani, B., Niaki, S. \&Aslanzade, S. (2017), Production-Inventory-Routing Coordination with Capacity and Time Window Constraints for Perishable Products: Heuristic and Meta-heuristic Algorithms. Journal of Cleaner Production;

[21]Yan, S., Chu, J. C., Hsiao, F.-Y. \& Huang, H.-J. (2015), A Planning Model and Solution Algorithm for Multi-Trip Split-Delivery Vehicle Routing and Scheduling Problems with Time Windows. Computers \& Industrial Engineering, 87, 383-393; [22]Zhang, Y., Snyder, L. V., Qi, M. \& Miao, L. (2016), A Heterogeneous Reliable Location Model with Risk Pooling under Supply Disruptions. Transportation Research Part B: Methodological, 83, 151-178;

[23]Zhong, Y. \& Cole, M. H. (2005), A Vehicle Routing Problem with Backhauls and Time Windows: A Guided Local Search Solution. Transportation Research Part E: Logistics and Transportation Review,41(2), 131-144. 